Classification

Physics Abstracts

$61.70-61.80-61.80 \mathrm{~F}-66.30$

\title{
Etude expérimentale de la dynamique de croissance de défauts étendus, sous irradiation électronique à haute énergie, dans le tellurure de cadmium
}

\author{
A. M. Gué et A. Mazel \\ Laboratoire d'Optique Electronique du C.N.R.S., 29 rue J. Marvig, 31055 Toulouse Cedex, France
}

(Reçu le 24 mars 1987, révisé le 11 septembre 1987, accepté le 21 septembre 1987)

\begin{abstract}
Résumé. - Les travaux que nous présentons ici apportent une contribution à l'étude des défauts étendus dans le CdTe obtenus après irradiation avec des électrons dont l'énergie est très nettement supérieure aux seuils de déplacement du cadmium et du tellure. Lors de ces irradiations, les atomes déplacés par les électrons incidents s'agglomèrent en positions interstitielles et forment des boucles de dislocation. Trois paramètres expérimentaux semblent avoir une importance primordiale : la tension accélératrice, la température et la dose. Il est mis en évidence qu'une élévation de tension favorise la formation en plus grand nombre des boucles. Les effets thermiques se manifestent par l'apparition d'amas de taille plus importante et de moindre densité lorsque la température croît. Lorsque le temps d'exposition au faisceau électronique est suffisamment long, une nouvelle génération d'agglomérats apparaît : ceux-ci sont constitués de lacunes.
\end{abstract}

\begin{abstract}
Some results of the study of extended defects observed in CdTe after irradiation with electrons of energy considerably higher than the threshold values for displacement of cadmium and tellurium are reported in this paper. During irradiation, the displaced atoms cluster and interstitial dislocation loops appear. This effect seems to be particularly sensitive to three experimental parameters : the accelerating voltage, the dose and the specimen temperature. It is shown that the density of defects becomes greater when the accelerating voltage is raised. Due to thermal effects, the size of the loops increases and their number decreases when the specimen temperature is increased. When the irradiation time is long enough, another kind of defects due to vacancies can be observed.
\end{abstract}

\section{Introduction.}

Le tellurure de cadmium [1] suscite depuis de nombreuses années un très grand intérêt, notamment sous la forme de son alliage ternaire $\mathrm{Hg}_{x} \mathrm{Cd}_{1-x} \mathrm{Te}$. Les applications visées concernent principalement les domaines suivants :

- la détection dans l'infrarouge [2],

- l'imagerie infrarouge [3],

- la détection $X$ et gamma [4].

Le développement des technologies afférentes à la réalisation de ces composants dépend principalement aujourd'hui de deux facteurs :

- la maîtrise de la croissance en couches minces,

- la maîtrise du dopage $[5,6]$.

De nombreux travaux antérieurs $[7,8]$ consacrés à ce sujet ont mis en exergue le rôle joué dans ce matériau par la présence de défauts ponctuels et étendus. Pour mieux comprendre ces problèmes et ceux liés aux défauts présents après la croissance du matériau ou à la suite des traitements de recuit, plusieurs auteurs ont utilisé les effets des rayonnements [9-11]. Ce type de procédé expérimental permet de caractériser, entre autres :

- la nature des différents défauts,

- leurs énergies de formation,

- les paramètres de diffusion des défauts ponctuels,

- les énergies mises en jeu lors de leur agglomération.

Sur le plan de l'interprétation, de nombreux points restent encore obscurs. En particulier, quels sont les mécanismes précis de création et d'interaction des défauts créés par le rayonnement incident?

L'étude par microscopie électronique des défauts créés par irradiation aux électrons, que nous proposons ici, s'inscrit parfaitement dans ce cadre et tente d'apporter dans une certaine mesure des réponses à ces problèmes. 


\section{Conditions expérimentales.}

Le CdTe utilisé a été élaboré par la technique THM (Travelling Heater Method) mise au point au Laboratoire de Physique des Solides de Meudon. Ce procédé permet d'obtenir un matériau monocristallin sur de larges zones, et dont la teneur en impuretés est faible $\left(<10^{14} / \mathrm{cm}^{3}\right)[12,13]$.

D'une manière générale, le clivage se faisant naturellement suivant des plans $\{110\}$, la normale aux lames cristallines ne s'écarte pas de plus de quelques degrés de la direction cristallographique $\langle 110\rangle$.

Les irradiations s'opèrent dans le microscope électronique $3 \mathrm{MV}$ de Toulouse muni d'un porteobjet inclinable $\left( \pm 20^{\circ}\right)$ permettant d'élever la température de l'échantillon en cours d'irradiation. Le flux du faisceau électronique est mesuré au moyen d'une cage de Faraday placée dans la chambre d'observation du microscope. Nous avons pu faire varier celui-ci entre $4 \times 10^{18}$ et $2 \times 10^{19}$ électrons/ $\mathrm{cm}^{2} . \mathrm{s}$.

Les résultats «bruts» se présentent sous deux formes :

- d'une part, des photographies prises en cours d'irradiation permettant d'observer la formation et la croissance des défauts étendus ;

- d'autre part, des clichés obtenus après irradiation avec un microscope électronique à basse tension (JEM 200 CX) utilisé pour la caractérisation cristallographique de ces défauts.

Le principal avantage de notre approche est de pouvoir suivre directement l'évolution des défauts créés par l'irradiation.

Compte tenu de la résolution des microscopes utilisés, les amas résultant de l'agglomération de ces défauts ne deviennent observables que lorsqu'ils atteignent une taille suffisante (diamètre de l'ordre de $50 \AA$ en général). En outre, étant donné la grande mobilité des atomes interstitiels, les effets des surfaces sur les concentrations et tailles des défauts ponctuels et étendus ne peuvent être ignorés et les comportements observés ne peuvent être assimilés à ceux du matériau massif que moyennant certaines précautions.

\section{Résultats expérimentaux.}

L'action du faisceau électronique dont la densité de flux est généralement de $6 \times 10^{18} \mathrm{e} / \mathrm{cm}^{2} . s$, se manifeste par l'apparition très rapide de "points noirs " dont le diamètre est de l'ordre de 40-50 $\AA$. Ces points noirs croissent et présentent au bout de quelques minutes le contraste caractéristique de boucles de dislocation (Fig. 1). Des mesures effectuées par stéréoscopie ont montré que, dans ces conditions, les défauts sont localisés généralement dans des zones proches de la surface exposée au faisceau. Lorsque l'irradiation est prolongée au-delà de 30 à 50 min (suivant les conditions expérimentales), un nombre très important de nouveaux défauts se forment entre les boucles déjà existantes ainsi que dans les zones minces. Ces amas, qui présentent peu à peu le contraste de boucles de dislocation, sont beaucoup plus denses que leurs analogues interstitielles et leur croissance semble plus lente. Bien que n'ayant pas caractérisé ces défauts, nous pouvons penser, par analogie avec les travaux de Hirata et Kiritani [14], que ces amas sont constitués de lacunes.

Trois paramètres expérimentaux ont une influence prépondérante sur les résultats obtenus : il s'agit de l'énergie des électrons, de la dose totale reçue par l'échantillon et enfin de la température à laquelle s'est effectuée l'irradiation.

3.1 Caractéristiques CRISTAllographiQues. Une étude de contraste effectuée à l'aide d'un microscope électronique à basse tension a permis de mettre en évidence la nature cristallographique des défauts étendus $[15,16]$. Nous rappellerons ici seulement que ceux-ci s'avèrent être des boucles de dislocations dont la forme est circulaire. Ces boucles sont constituées d'atomes interstitiels et leurs vecteurs de Burgers sont du type $a / 3\langle 111\rangle, a / 2\langle 110\rangle$ et $a\langle 100\rangle$.

Ces caractéristiques ont été vérifiées quelles que soient les valeurs des paramètres expérimentaux.

Cependant, il est à noter que les différents types de boucles de dislocation n'apparaissent pas dans les mêmes proportions. Ainsi les boucles de type « $\langle 111\rangle$ " sont les plus fréquentes (en moyenne $80 \%)$ alors que les boucles de type « $\langle 100\rangle »$ sont en très petit nombre sur une même zone. Le tableau I donne un exemple de la répartition des boucles suivant leur nature pour trois échantillons irradiés à même tension et même température mais avec des densités de flux différentes. D'une manière générale, il semble donc que l'agglomération des atomes interstitiels se fait dans des plans de type $\{111\}$. Ceci est en accord avec les résultats de Yoshiie et al. [17]. Toutefois, il est à noter que ces auteurs n'ont pas observé, lors de leurs travaux, de boucles de vecteur de Burgers $a\langle 100\rangle$.

3.2 Densité ET TAILle DES DÉFAuTS CRÉÉs. Ces deux caractéristiques sont intimement liées et varient, en sens inverse l'une de l'autre. Il est nécessaire ici d'envisager séparément l'influence des différents paramètres expérimentaux.

3.2.1 Energie des électrons. - La figure 1 représente l'évolution d'une zone sous l'impact des électrons $\left(\Phi=6 \times 10^{18}\right.$ électrons $/ \mathrm{cm}^{2} . \mathrm{s}, \quad T=380 \mathrm{~K}, \quad V=$ $1 \mathrm{MV}$ ). Son observation minutieuse nous permet de constater que les boucles nettement visibles après 3 min d'irradiation ne varient plus ensuite en nombre 

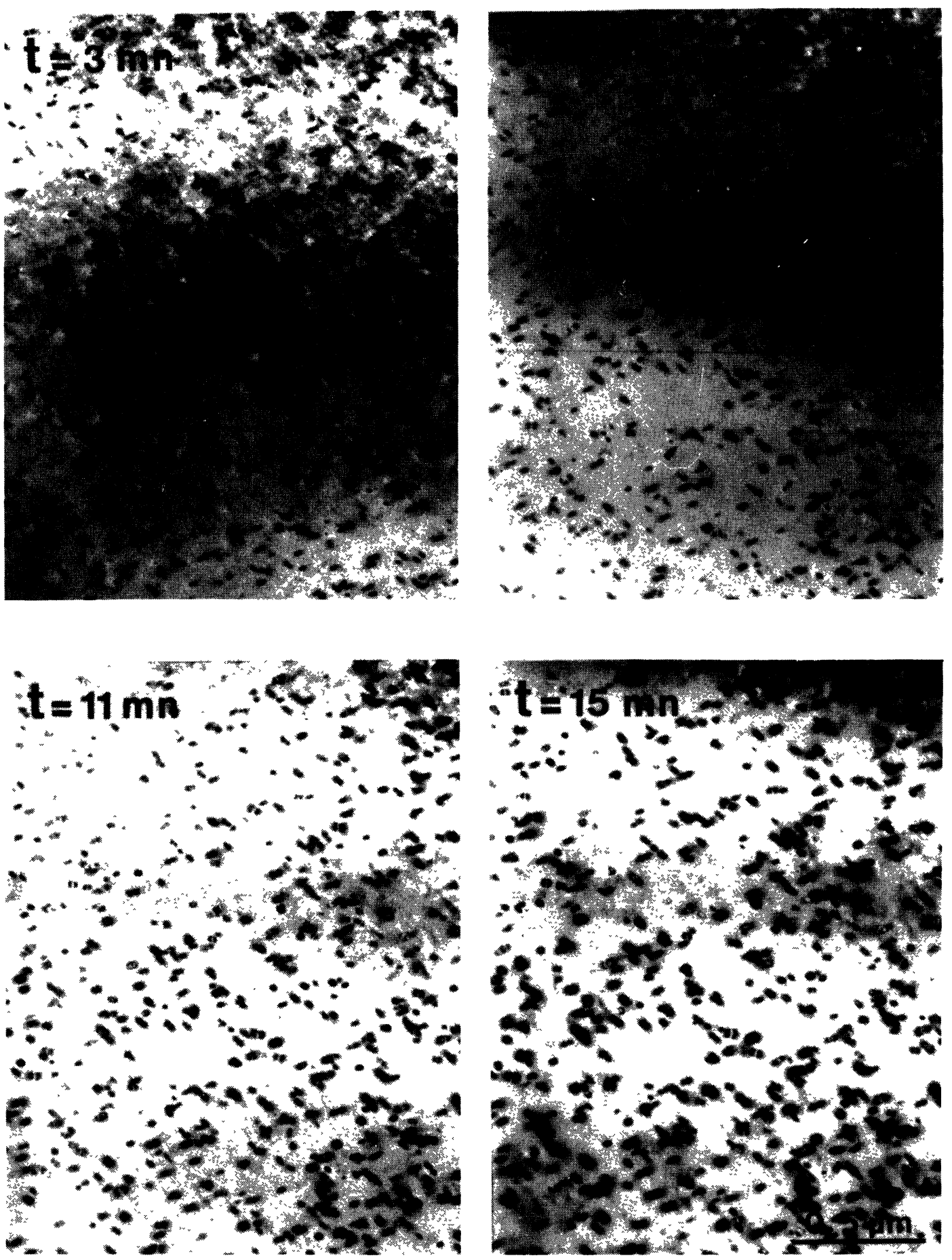

Fig. 1. - Evolution d'une zone irradiée dans les conditions suivantes: $V=1 \mathrm{MV}, \quad \Phi=6 \times 10^{18} \mathrm{e} / \mathrm{cm}^{2} . \mathrm{s}$, $T=380 \mathrm{~K}$.

[Evolution of an area irradiated under the following conditions : $V=1 \mathrm{MV}, \Phi=6 \times 10^{18} \mathrm{e} / \mathrm{cm}^{2} . \mathrm{s}, T=380 \mathrm{~K}$.]

mais seulement en taille. La courbe représentée sur la figure 2 , relative à une zone irradiée à $1,5 \mathrm{MV}$ et à une température de $420 \mathrm{~K}$, rend compte de ce résultat. En effet, l'on constate que la densité des boucles ne croît pratiquement plus au-delà de la 6-ième minute d'exposition sous le faisceau d'électrons. Cet effet a été régulièrement observé à $1 \mathrm{MV}$ et 1,5 MV. Dans cette gamme de tension, succède à la courte phase de germination une phase de grossissement où seule la taille des agglomérats varie.

A $2 \mathrm{MV}$, l'irradiation fait apparaître un enchevêtrement de boucles comme il est possible de l'observer sur la figure 3. La trop forte densité des amas rend l'exploitation mal aisée. Cependant, une comparaison avec des zones irradiées à 1,5 MV permet de constater que le nombre de défauts est 
Tableau I. - Répartition des boucles de dislocation en fonction de leurs caractéristiques cristallographiques. [Relative densities of dislocation loops versus their cristallographic features.]

\begin{tabular}{|c|c|c|c|c|}
\hline $\begin{array}{c}\text { Type des } \\
\text { boucles }\end{array}$ & $\begin{array}{c}\Phi=9,9 \times 10^{19} \mathrm{e} / \mathrm{cm}^{2} . \mathrm{s} \\
T=380 \mathrm{~K} \\
\text { Echantillon A }\end{array}$ & $\begin{array}{c}\Phi=6 \times 10^{18} \mathrm{e} / \mathrm{cm}^{2} . \mathrm{s} \\
T=380 \mathrm{~K} \\
\text { Echantillon B }\end{array}$ & $\begin{array}{c}\Phi=6 \times 10^{18} \mathrm{e} / \mathrm{cm}^{2} . \mathrm{s} \\
T=380 \mathrm{~K} \\
\text { Echantillon C }\end{array}$ & $\begin{array}{l}\text { Valeur } \\
\text { moyenne }\end{array}$ \\
\hline$a / 3\langle 111\rangle$ & $97,5 \%$ & $67,5 \%$ & $75,2 \%$ & $80,0 \%$ \\
\hline$a / 2\langle 011\rangle$ & \multirow{2}{*}{$2,5 \%$} & $24,6 \%$ & $14,5 \%$ & $13,8 \%$ \\
\hline$a\langle 001\rangle$ & & $7,7 \%$ & $10,3 \%$ & $6,7 \%$ \\
\hline
\end{tabular}

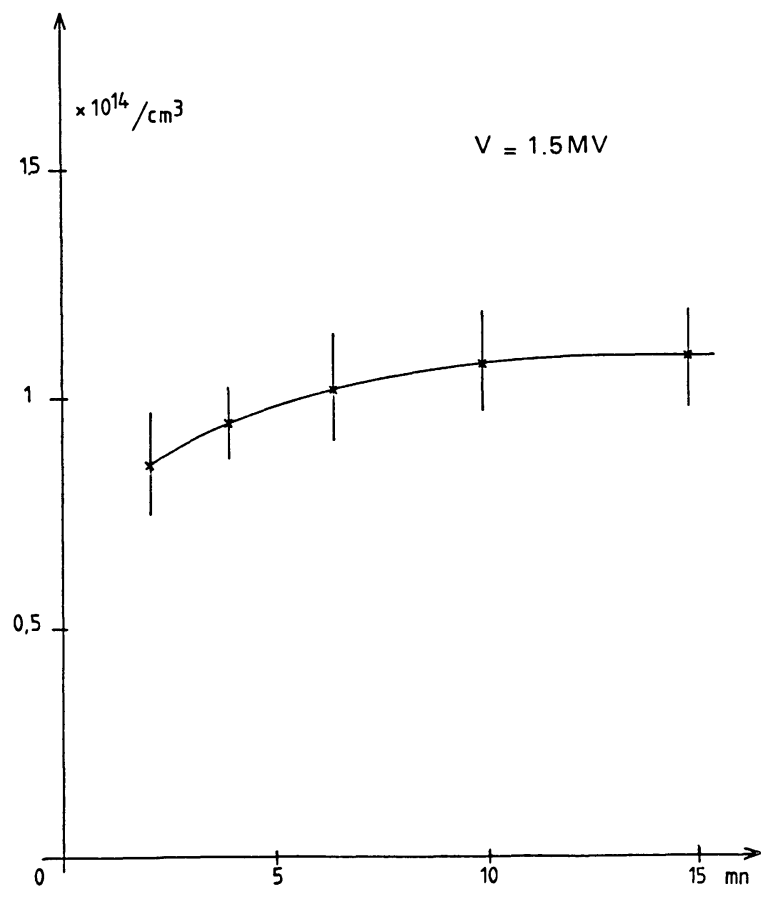

Fig. 2. - Densité de boucles créées en fonction du temps d'irradiation.

[Density of loops as a function of irradiation time.]

très nettement supérieur. Si l'on étudie plus précisément le comportement de la densité de boucles en fonction de l'énergie des électrons incidents, on constate (Tab. II) que le nombre de boucles ne croît pas de manière significative entre 1 et $1,5 \mathrm{MV}$. Par

Tableau II. - Densité de boucles créées en fonction de la tension accélératrice.

[Density of dislocation loops versus the accelerating voltage.]

\begin{tabular}{|c|c|c|c|}
\hline$V$ & $1 \mathrm{MV}$ & $1,5 \mathrm{MV}$ & $2 \mathrm{MV}$ \\
\hline $\mathrm{Nb}$ de boucles $/ \mathrm{cm}^{3}$ & $2,45 \times 10^{14}$ & $3,3 \times 10^{14}$ & $14,3 \times 10^{14}$ \\
\hline
\end{tabular}

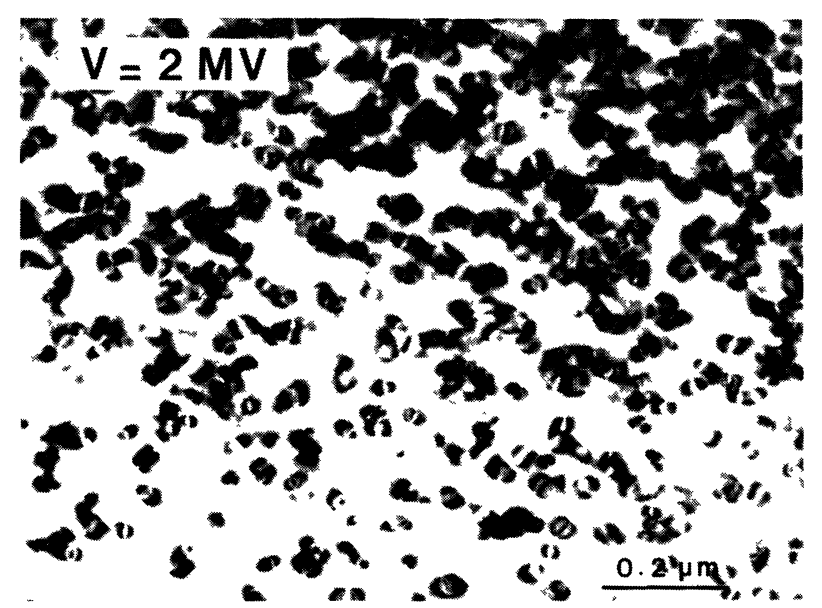

Fig. 3. - Exemple d'une zone irradiée à $2 \mathrm{MV}$, $\Phi=6 \times 10^{18} \mathrm{e} / \mathrm{cm}^{2} . \mathrm{s}, T=380 \mathrm{~K}, t=15 \mathrm{~min}$.

[Micrography of a specimen irradiated at $2 \mathrm{MV}$, the other experimental conditions are : $\Phi=6 \times 10^{18} \mathrm{e} / \mathrm{cm}^{2} . \mathrm{s}, T=$ $380 \mathrm{~K}, t=15 \mathrm{~min}$.

contre à $2 \mathrm{MV}$ il y a une très forte augmentation du nombre de défauts alors que le rayon moyen des boucles formées reste pratiquement inchangé. Il semble donc qu'aux fortes énergies un élément nouveau intervienne.

3.2.2 Température d'irradiation. - Afin de mettre en évidence les effets thermiques, des irradiations ont été effectuées dans des domaines de température très différents (de 18 à $500 \mathrm{~K}$ ).

La photographie de la figure 4 illustre le cas d'une irradiation à très basse température ; la zone irradiée présente une pigmentation très dense constituée d'une multitude de points noirs qui souvent ne sont pas résolus. Ces points noirs sont donc la preuve que l'agglomération des défauts ponctuels n'a pas été complètement interdite et qu'une forme de diffusion est intervenue.

Par ailleurs, les expériences effectuées à température ambiante et aux environ de $400 \mathrm{~K}$ ont montré la 


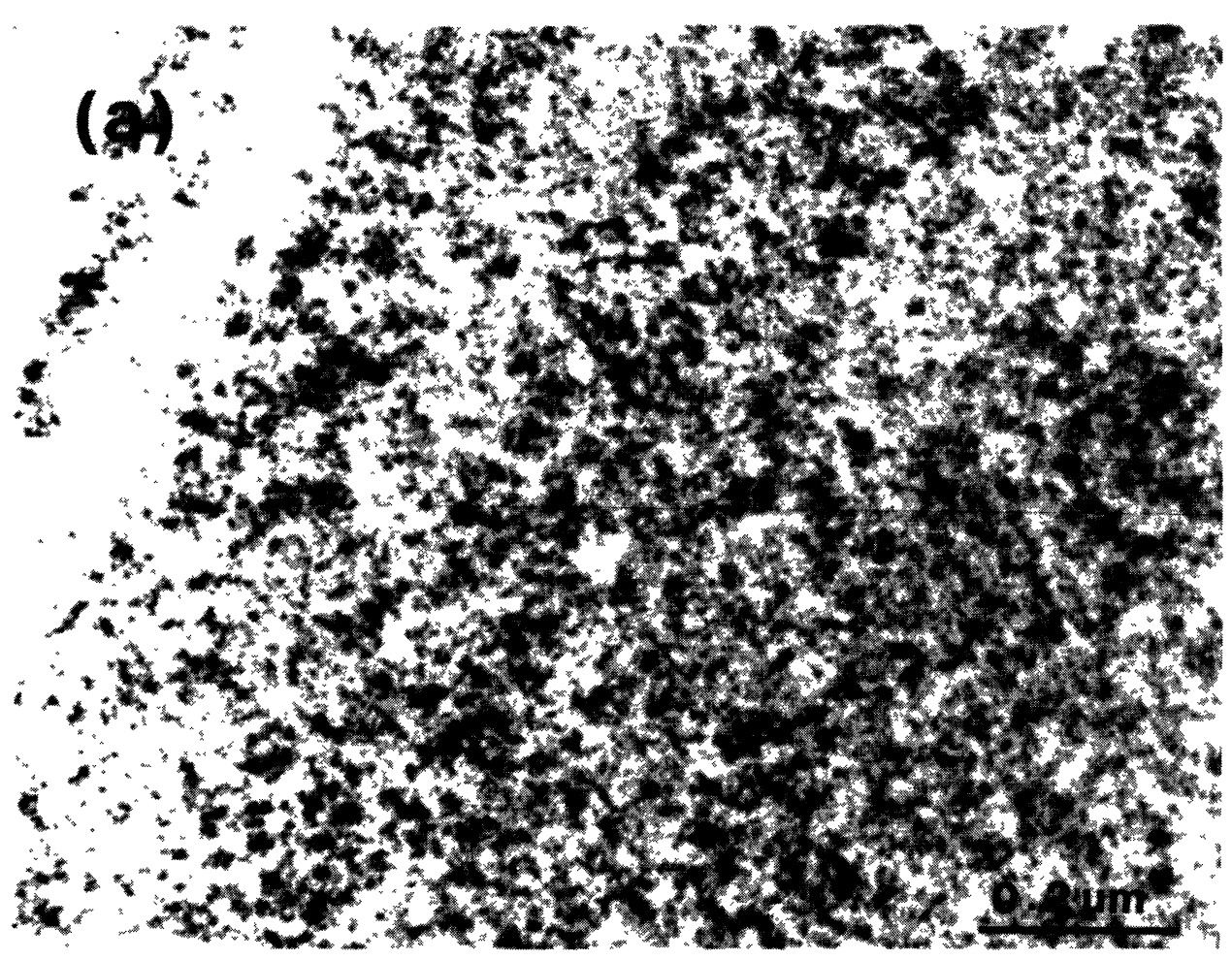

Fig. 4. - Zone irradiée à $18 \mathrm{~K}$. Les autres conditions expérimentales sont : $\Phi=6 \times 10^{18} \mathrm{e} / \mathrm{cm}^{2} . \mathrm{s}, V=1 \mathrm{MV}$, $t=15 \min$.

[Micrography of an area irradiated at $18 \mathrm{~K}$. The other experimental conditions are : $\Phi=6 \times 10^{18} \mathrm{e} / \mathrm{cm}^{2} . \mathrm{s}$, $V=1 \mathrm{MV}, t=15 \mathrm{~min}$.

grande sensibilité des résultats aux variations de la température d'irradiation.

La figure 5 présente deux zones irradiées dans les mêmes conditions, c'est-à-dire à des tensions accélératrices, des flux et doses identiques. Les expériences ont été effectuées en excitant des réflexions de même type, mais les températures sont différentes. D'après les résultats obtenus, il apparaît que la taille des amas formés à $420 \mathrm{~K}$ est supérieure à celle des boucles formées à $380 \mathrm{~K}$ et que leur densité est moindre. Le rayon moyen qui est de $120 \AA$ à $380 \mathrm{~K}$ devient, en effet, $165 \AA$ à $420 \mathrm{~K}$.

Au cours des irradiations effectuées à $480 \mathrm{~K}$, nous avons constaté que toute agglomération était rendue impossible et que la zone irradiée restait vierge de tout agglomérat. Ceci est également en accord avec les résultats de Yoshiie et al. [17].

On peut donc conclure de l'ensemble de ces expériences que lorsque la température s'élève, le rayon moyen des défauts augmente alors que leur densité diminue. Le tableau III donne la taille moyenne des boucles pour diverses valeurs de la température. Si l'on étudie plus précisément quelle est la répartition des défauts en fonction de leur diamètre, l'on est conduit aux remarques suivantes (Fig. 6).

- La distribution s'écarte progressivement des
Tableau III. - Taille moyenne des boucles en fonction de la température.

[Mean size of the loops versus temperature.]

\begin{tabular}{|c|c|c|c|}
\hline$T=100 \mathrm{~K}$ & $T=380 \mathrm{~K}$ & $T=392 \mathrm{~K}$ & $T=420 \mathrm{~K}$ \\
\hline $180 \AA$ & $240 \AA$ & $260 \AA$ & $330 \AA$ \\
\hline
\end{tabular}

rayons de faibles valeurs lorsque la température s'élève.

- Entre 300 et $380 \mathrm{~K}$, elle semble plus étendue vers les rayons supérieurs à la valeur moyenne et n'est donc pas symétrique. Par contre, à partir de $390 \mathrm{~K}$, la dimension des amas la plus probable correspond à la valeur moyenne.

3.2.3 Dose. - L'influence de la dose apparaît sans ambiguïté sur les quatre clichés de la figure 1 qui montrent que la taille des amas augmente lorsque l'irradiation se prolonge. Nous avons reporté sur la figure 7 , en échelle logarithmique, les variations du rayon moyen des boucles en fonction du temps d'irradiation. On obtient dans chaque cas une droite dont la pente est de l'ordre de $1 / 3$ lorsque la température n'excède pas $380 \mathrm{~K}$ et $1 / 2$ à $420 \mathrm{~K}$. Nous pouvons donc en conclure que la croissance des 

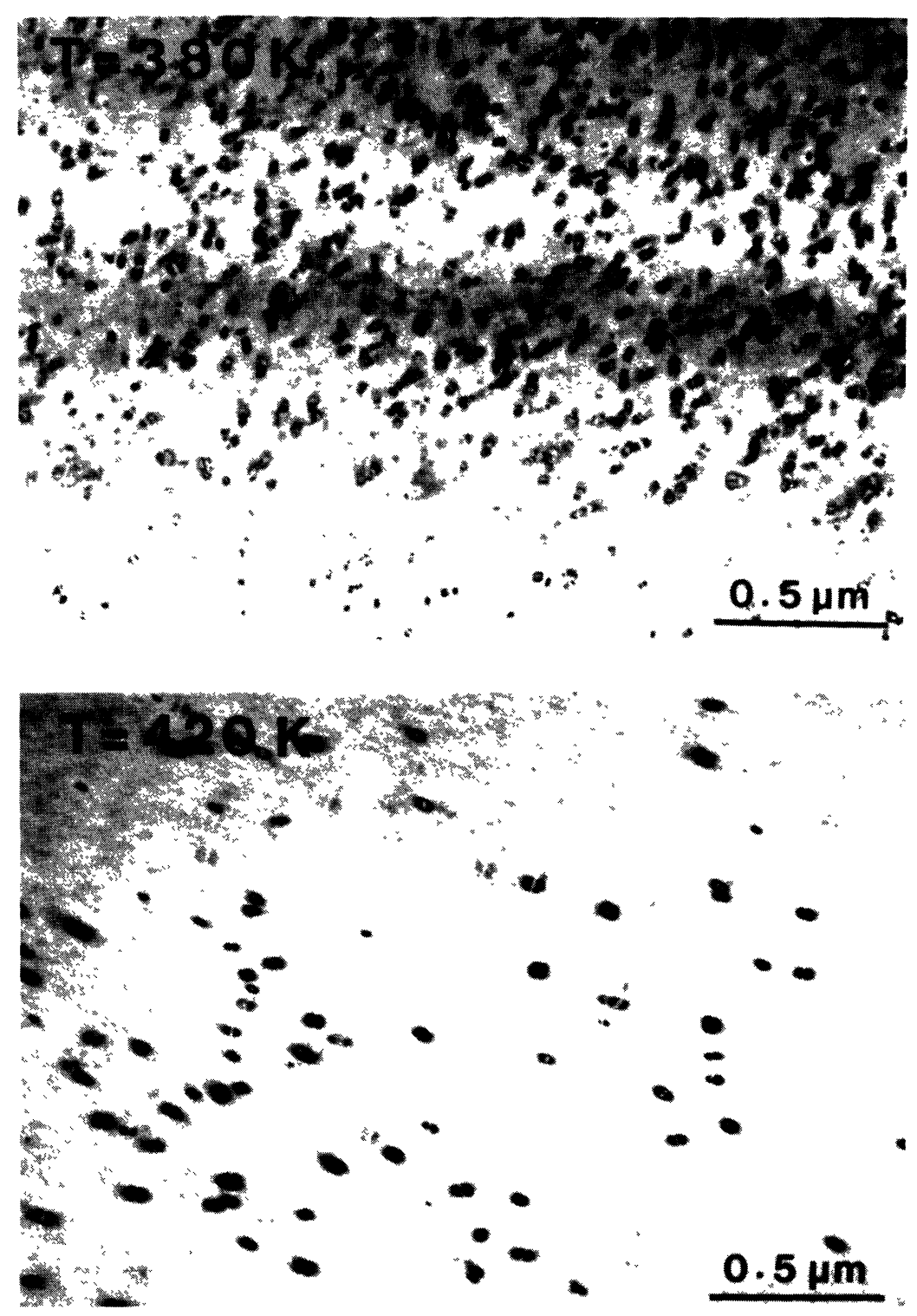

Fig. 5. - Influence de la température. a) Zone irradiée à $T=380 \mathrm{~K}, V=1,5 \mathrm{MV}$ et $\Phi=6 \times 10^{18} \mathrm{e} / \mathrm{cm}^{2}$. $\mathrm{s}$ pendant 15 min. b) Zone irradiée à $T=420 \mathrm{~K}$, les autres paramètres ayant des valeurs identiques. On peut noter la plus faible densité et la taille supérieure des boucles formées à $420 \mathrm{~K}$.

[Temperature influence. a) Specimen irradiated at $T=380 \mathrm{~K}, V=1.5 \mathrm{MV}$ and $\Phi=6 \times 10^{18} \mathrm{e} / \mathrm{cm}^{2}$.s during $15 \mathrm{~min}$. b) Specimen irradiated at $T=420 \mathrm{~K}$, the other parameters being the same. It can be noticed that the density of the loops is lower than at $380 \mathrm{~K}$ and that their size is bigger.]

amas se fait en $t^{x}$ où $x$ est environ $1 / 3$ puis tend vers $1 / 2$ lorsque la température s'élève.

\section{Conclusion.}

Dans ce travail, nous nous sommes attachés à examiner de manière assez systématique l'influence de divers paramètres expérimentaux et plus particulièrement de la température sur les défauts créés par les électrons dans le CdTe.

Ces résultats expérimentaux nous amènent d'ores et déjà à un certain nombre de commentaires.

Le déplacement des atomes interstitiels créés par les particules incidentes permet la germination puis la formation d'amas. Ceux-ci, lorsqu'ils atteignent une certaine taille deviennent fixes et constituent des pièges pour les interstitiels non agglomérés. Dans une gamme d'énergies ne dépassant pas $1,5 \mathrm{MeV}$, nous pouvons constater que cette phase de germination est brève et qu'il lui succède une phase de grossissement où, à nombre de boucles de dislocation défini, leur taille grandit. Ceci nous permet donc de penser que nous sommes, à ces tensions là, en présence d'une germination homogène.

Par contre, nous avons pu constater à partir du tableau II, que lors d'irradiations à des tensions 

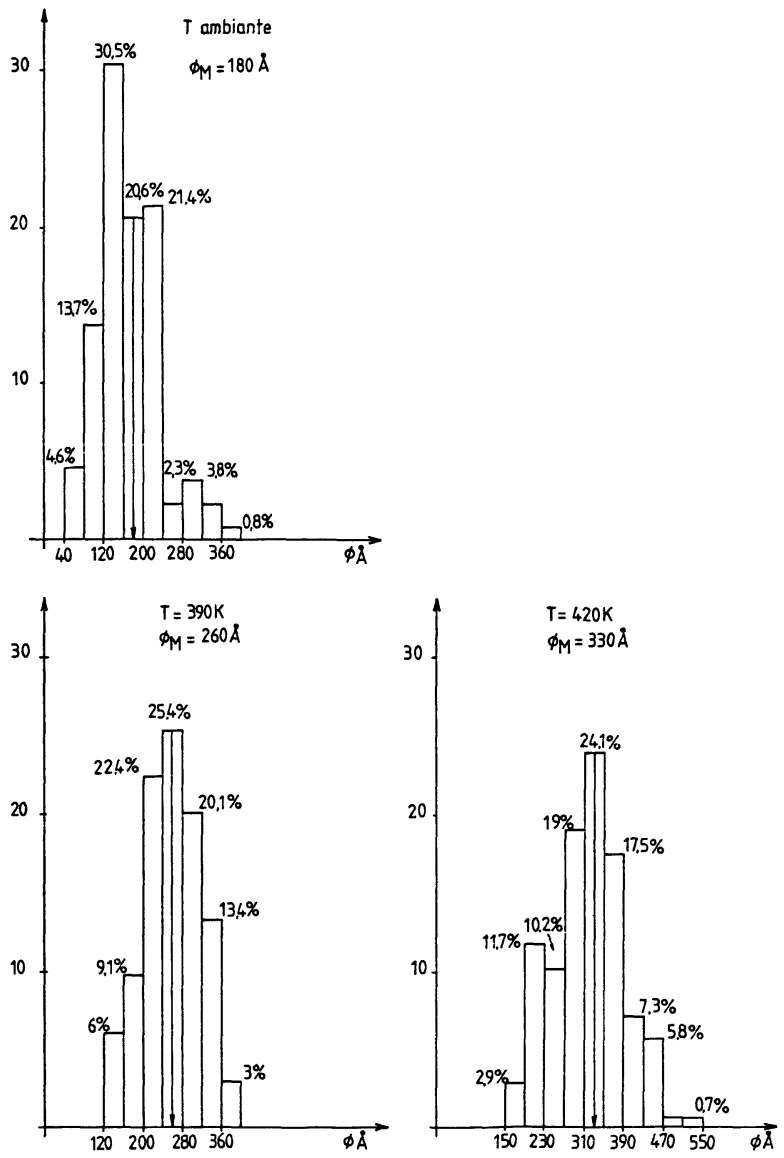

Fig. 6. - Répartition des boucles d'une même zone en fonction de leur taille pour trois températures d'irradiation. La valeur moyenne est indiquée par une flèche.

[Size distribution of loops for three irradiation temperatures. The mean values are indicated by an arrow.] accélératrices supérieures à 1,5 MV, le nombre de boucles créées croissait anormalement. Si l'on cherche dans les probabilités de déplacement des atomes une explication à ce phénomène [18-20], on constate que la section efficace de déplacement ne rend pas compte d'une variation aussi brutale même si l'on considère la possibilité de cascades (Fig. 8). C'est donc qu'un nouveau processus intervient. L'hypothèse d'une germination hétérogène est séduisante car elle prévoit la création d'un très grand nombre de boucles de faibles dimensions qui peuvent s'agglomérer par montée conservative lorsque leur densité est importante [21]. Dans ce cas, la phase de germination peut se prolonger au-delà des tous premiers instants de l'irradiation.

A travers ces diverses observations, il apparaît sans équivoque que le nombre de défauts créés est d'autant plus important que l'énergie des électrons est élevée.

La température ayant une influence considérable sur les tailles et densité des agglomérats, on peut penser que les mécanismes de migration sont principalement liés à une diffusion activée thermiquement. Cependant, nous avons pu constater sur la photo de la figure 5 que, même à des températures très basses, une forme de diffusion avait permis aux interstitiels de former des amas de dimension toutefois réduite. La faible taille de ces amas et leur densité importante montrent que cette migration se fait sur de courtes distances. Il est difficilement envisageable d'imputer ces déplacements atomiques à une diffusion thermique. Ainsi, il est possible de rapprocher ces phénomènes des mécanismes ather-

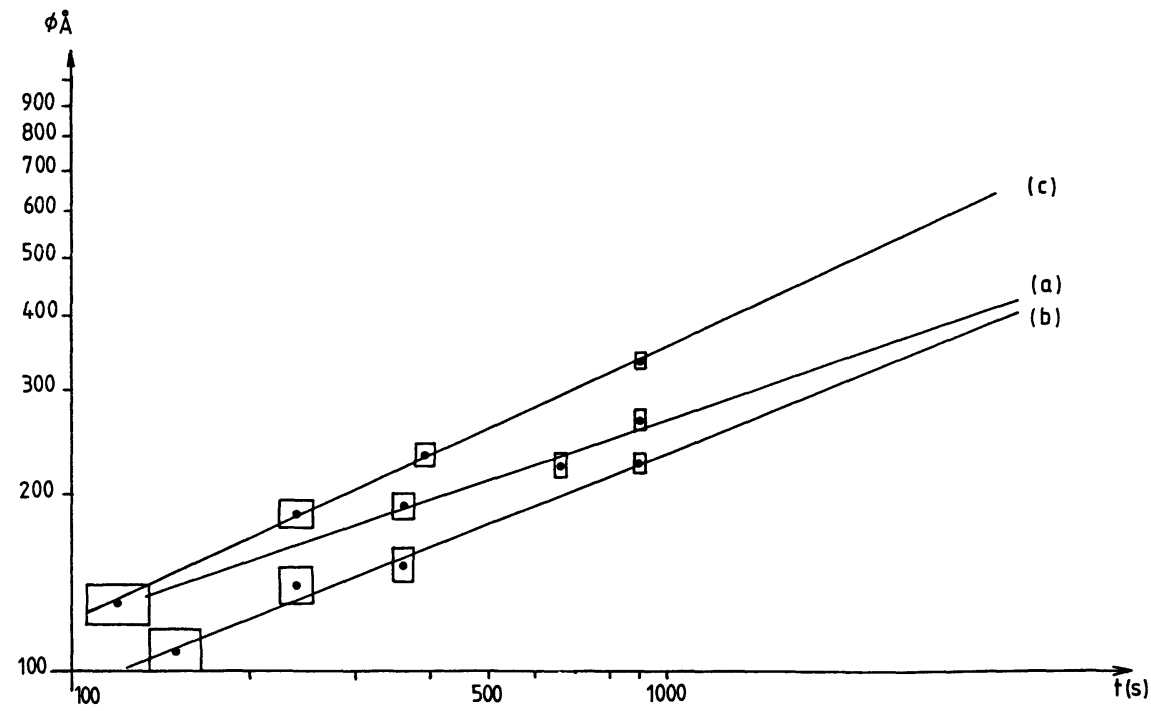

Fig. 7. - Variation du rayon moyen des boucles en fonction du temps d'irradiation pour différentes conditions expérimentales: a) $T=380 \mathrm{~K}, V=1 \mathrm{MV}, \Phi=6 \times 10^{18} \mathrm{e} / \mathrm{cm}^{2}$.s. La pente $x$ est $x=0,34 ;$ b) $T=380 \mathrm{~K}$, $V=1,5 \mathrm{MV}, \Phi=6 \times 10^{18} \mathrm{e} / \mathrm{cm}^{2}$.s. $x=0,4$; c) $T=420 \mathrm{~K}, V=1,5 \mathrm{MV}, \Phi=6 \times 10^{18} \mathrm{e} / \mathrm{cm}^{2} . \mathrm{s} . x=0,524$.

[Time dependence of the mean size of the loops under various irradiation conditions : a) $T=380 \mathrm{~K}, V=1 \mathrm{MV}$, $\Phi=6 \times 10^{18} \mathrm{e} / \mathrm{cm}^{2}$.s. The slope $x$ is $x=0.34$; b) $T=380 \mathrm{~K}, V=1.5 \mathrm{MV}, \Phi=6 \times 10^{18} \mathrm{e} / \mathrm{cm}^{2} . \mathrm{s} . x=0.4$; c) $\left.T=420 \mathrm{~K}, V=1.5 \mathrm{MV}, \Phi=6 \times 10^{18} \mathrm{e} / \mathrm{cm}^{2} \mathrm{~s} . x=0.524.\right]$ 


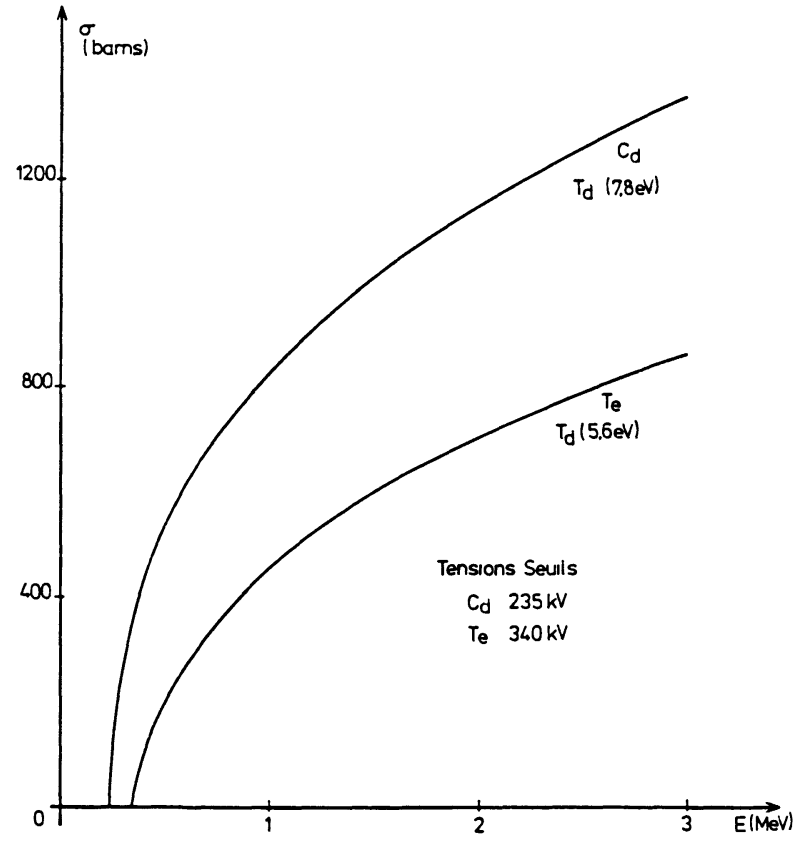

Fig. 8. - Sections efficaces de déplacement du cadmium et du tellure en fonction de l'énergie cinétique des électrons incidents calculées à partir des tables de Oen [18]. Les énergies seuil de déplacement utilisées sont celles déterminées par Bryant et al. [19, 20].

[Displacement cross-section of cadmium and tellurium as a function of the kinetic energy of electrons calculated with values determinated by Oen [18] and with threshold displacement energy given by Bryant et al. $[19,20]$.

miques, induits par l'irradiation, qui peuvent permettre la migration des atomes d'un composé semiconducteur $[22,23]$.

En ce qui concerne les caractéristiques cristallogra- phiques des boucles de dislocation interstitielles, nous avons vu que celles-ci se formaient de préférence par insertion des deux plans atomiques $\{111\}$ de natures différentes [17]. Ce résultat est différent de ceux obtenus dans le cas des composés III-V dont les propriétés structurales sont cependant identiques à celles du CdTe [24,25]. En effet, les composés III-V de structure zinc blende irradiés aux électrons présentent des boucles de dislocation dont le vecteur de Burgers est toujours de type $a / 2$ $\langle 110\rangle$. Le fait que des boucles fautées $\langle 111\rangle$ n'aient pas été observées dans ces matériaux trouve son explication dans les valeurs des énergies de faute d'empilement. Celles-ci sont, en général, largement supérieures à celles des composés II-VI [26]. En particulier, l'énergie de faute d'empilement de l'AsGa est 5 fois celle du CdTe. Pour expliquer une telle différence, il faut prendre en compte l'ionicité du cristal. Il a été démontré [26] que l'énergie de faute d'empilement est d'autant plus faible que le cristal présente un caractère ionique. Il apparaît évident que dans le cas des composés fortement ioniques comme le CdTe [26] les fautes d'empilement sont stabilisées et la formation de boucles $\langle 111\rangle$ favorisée.

\section{Remerciements.}

Les auteurs remercient vivement M. R. Triboulet du Laboratoire de Physique des Solides de Bellevue (C.N.R.S. Meudon) qui a bien voulu fournir les échantillons. Ils remercient également MM. D. Estève et F. Therez du L.A.A.S. (C.N.R.S. Toulouse) pour les fructueuses discussions scientifiques qu'ils ont eues ensemble.

\section{Bibliographie}

[1] Zanio, K., Semiconductors and Semimetals, Vol. 13, Cadmium Telluride (Academic Press) 1978.

[2] Kruse, P. W., Semiconductors and Semimetals, Vol. 18 (Academic Press) 1981.

[3] LaBaw, C., Proc. SPIE, Int. Soc. Opt. Eng. 430 (1983) 68

[4] Jones, L. T., Revue Phys. Appl. 12 (1977) 379.

[5] Donnelly, J. P., Foyt, H. G., Hinkley, E. D., Lindley, W. T. and Dimmock, J. O., Appl. Phys. Lett. 12 (1968) 9.

[6] Wu, S. Y. and Chоуке, W. J., J. Vac. Soc. Technol. 21 (1982) 1.

[7] Kalish, R., Deischer, M. and Schatz, G., J. Appl. Phys. 53 (1982) 9.

[8] Devlin, S. S., Physics and chemistry of II-VI Compounds (North-Holland Publishing Company-Amsterdam) 1967.

[9] Taguchi, T., Shirafuji, J. and InUishi, Y., Nucl. Inst. Meth. 150 (1978) 43.
[10] Caillot, M., Inst. Phys. Conf. Ser. n ${ }^{\circ} 23$ (1975).

[11] Watkins, G. D., Rad. Eff. 9 (1971) 105.

[12] Triboulet, R. and Marfaing, Y., J. Appl. Phys. 45 (1974) 6.

[13] Triboulet, R. and Marfaing, Y., J. Cryst. Growth $51 \mathrm{n}^{\circ} 1$ (1981).

[14] Hirata, M. and Kiritani, M., Proc. of the 7th Int. Conf. on H.V.E.M., Berkeley (1983).

[15] Gué, A. M. and MAzel, A., J. Cryst. Growth 72 (1985) 210.

[16] GuÉ, A. M., Thèse $3^{c}$ cycle Toulouse (1985).

[17] Yoshile, T., Iwanaga, H., Shibata, N., Suzuki, R., Ichihara, M., Takeuchi, S., Philos. Mag. A $47 \mathrm{n}^{\circ} 3$ (1983).

[18] OEN, O. S., ORNL 4887 (1973).

[19] Bryant, F. J. and Webster, E., Phys. Status Solidi 21 (1967) 315.

[20] Bryant, F. J., Cox, A. F. T. and Webster, E., J. Phys. C Ser. 21 (1968). 
[21] Bourret, A., Thèse d'Etat Grenoble (1970).

[22] Urban, K. and Seeger, A., Philos. Mag. 6 (1974) 1395.

[23] Bourgoin, J. C. and Corbett, J. W., Inst. Phys. Conf. Ser. 23 (1974) 149-163.

[24] Legros-de Mauduit, B., Lasserre, A. and ReyNAUD, F., Rad. Eff. 62 (1982) 111-115.
[25] Reynaud, F., Mazel, A., Legros de Mauduit, B. and Ajustron, F., Proc. of the 7th Int. Conf. on H.V.E.M. (1983).

[26] Takeuchi, S., Suzuki, K. and Maeda, K., Philos. Mag. A 50 (1984) 2. 